High-density lipoproteins in sepsis and septic shock: metabolism, actions, and therapeutic applications. Shock. 21:210-221.

18. Shiflett, A.M., Bishop, J.R., Pahwa, A., and Hajduk, S.L. 2005. Human high density lipoproteins are platforms for the assembly of multi-component innate immune complexes. J. Biol. Chem. 280:32578-32585

19. Rezaee, F., Casetta, B., Levels, J.H., Speijer, D., and Meijers, J.C. 2006. Proteomic analysis of high-density lipoprotein. Proteomics. 6:721-730.

20. Zheng, L., et al. 2005. Localization of nitration and chlorination sites on apolipoprotein A-I catalyzed by myeloperoxidase in human atheroma and associated oxidative impairment in ABCA1-dependent cholesterol efflux from macrophages. J. Biol. Chem. 280:38-47.

21. Shao, B., et al. 2006. Myeloperoxidase impairs ABCA1-dependent cholesterol efflux through methionine oxidation and site-specific tyrosine chlorination of apolipoprotein A-I. J. Biol. Chem. 281:9001-9004.
22. Heller, M., et al. 2005. Mass spectrometry-based analytical tools for the molecular protein characterization of human plasma lipoproteins. Proteomics. 5:2619-2630.

23. Karlsson, H., Leanderson, P., Tagesson, C., and Lindahl, M. 2005. Lipoproteomics II: mapping of proteins in high-density lipoprotein using two-dimensional gel electrophoresis and mass spectrometry. Proteomics. 5:1431-1445.

24. Hortin, G.L., Shen, R.F., Martin, B.M., and Remaley, A.T. 2006. Diverse range of small peptides associated with high-density lipoprotein. Biochem. Biophys. Res. Commun. 340:909-915.

25. Hamilton, K.K., Zhao, J., and Sims, P.J. 1993. Interaction between apolipoproteins A-I and A-II and the membrane attack complex of complement. Affinity of the apoproteins for polymeric C9.J. Biol. Chem. 268:3632-3638.

26. Mineo, C., Deguchi, H., Griffin, J.H., and Shaul, P.W. 2006. Endothelial and antithrombotic actions of HDL. Circ. Res. 98:1352-1364.

27. Asztalos, B.F., et al. 2004. High-density lipoprotein subpopulation profile and coronary heart disease prevalence in male participants of the Framingham Offspring Study. Arterioscler. Thromb. Vasc. Biol. 24:2181-2187.

28. Freedman, D.S., et al. 2004. Sex and age differences in lipoprotein subclasses measured by nuclear magnetic resonance spectroscopy: the Framingham Study. Clin. Chem. 50:1189-1200.

29. Eriksson, M., Carlson, L.A., Miettinen, T.A., and Angelin, B. 1999. Stimulation of fecal steroid excretion after infusion of recombinant proapolipoprotein A-I. Potential reverse cholesterol transport in humans. Circulation. 100:594-598.

30. Parks, E.J., and Hellerstein, M.K. 2006. Thematic review series: patient-oriented research. Recent advances in liver triacylglycerol and fatty acid metabolism using stable isotope labeling techniques. J. Lipid Res. 47:1651-1660.

31. Ansell, B.J., Watson, K.E., Fogelman, A.M., Navab, M., and Fonarow, G.C. 2005. High-density lipoprotein function recent advances. J. Am. Coll. Cardiol. 46:1792-1798.

\title{
Autoantibody selection and production in early human life
}

\author{
Eric Meffre and Jane E. Salmon
}

Hospital for Special Surgery, Weill Medical College of Cornell University, New York, New York, USA.

\begin{abstract}
Natural antibodies are autoreactive/polyreactive antibodies believed to be secreted in the absence of xenoantigens. The origin and functional role of this limited and selective autoimmunity are not clear, nor is the specificity and range of autoantigens that drive the development of $B$ cells producing natural antibodies. In this issue of the JCI, Merbl et al. report that in utero, humans generate natural IgM and IgA antibodies that recognize a uniform set of autoantigens (see the related article beginning on page 712), some of which are associated with autoimmune diseases. The authors postulate that this "autoimmunity" at birth favors the emergence of autoimmune diseases in later life. We present a molecular basis for the limited and common repertoire of antibodies produced by fetal $B$ cells, which may be distinct from the abnormalities in $B$ cell development described in patients with autoimmune diseases.
\end{abstract}

$\mathrm{B}$ cell tolerance checkpoints remove many developing self-reactive $\mathrm{B}$ cells from the adult repertoire, yet the presence of certain autoreactive B cells in normal individuals is revealed by the identification of serum autoantibodies referred to as natural antibodies $(1,2)$. Most natural antibodies are low-affinity IgM autoantibodies that are

Nonstandard abbreviations used: BCR, B cell receptor; CDR3, complementarity-determining region 3; D, diversity; $\mathrm{H}$, heavy chain; IgH, Ig heavy chain; J, joining; NMHC-II, nonmuscle myosin heavy chain type II; TDT, terminal deoxynucleotidyl transferase; V, variable; XLA, $\mathrm{X}$-linked agammaglobulinemia.

Conflict of interest: The authors have declared that no conflict of interest exists.

Citation for this article: J. Clin. Invest. 117:598-601 (2007). doi:10.1172/JCI31578. often polyreactive and bind to a broad range of self antigens $(2,3)$. In mice, natural antibody-secreting $B$ cells belong to a specific B cell subpopulation called B-1 cells, which preferentially reside in the peritoneal cavity (4). The fact that these peripheral autoreactive B cells are positively selected by self antigens seems paradoxical to $B$ cell tolerance, but raises the possibility that autoantibodies may in some cases perform useful functions (5). In humans, the origin of natural antibodies and the autoantigens that they recognize remain to be characterized. In this issue of the JCI, Merbl et al. (6), using an antigen microarray, identified specific autoantigens recognized by natural IgM and, to a lesser extent, IgA antibodies produced during human fetal and neonatal life (6). In addition, they found that autoantigens recognized by serum IgMs from newborns were remarkably similar among individuals, suggesting that in the absence of xenoantigens, specific autoantigens may select and stimulate autoreactive B cells to produce self-reactive natural IgM antibodies in fetuses (6). In contrast, IgM and IgA antibodies from mothers showed an antigenic recognition pattern different from that of their newborns. Furthermore, IgM and IgA recognition patterns were extremely diverse among the mothers studied, reflecting the distinct immunological histories of each individual. However, encounters with some common pathogens, such as Gram-negative bacteria, stimulated the production of antiLPS antibodies in all mothers (6).

\section{Potential origins of common natural antibody reactivity at birth}

What might account for the recognition of the same autoantigens by different individuals during early human development? Antibodies are generated by random recombination of Ig variable $(\mathrm{V})$, diversity (D), and joining $(\mathrm{J})$ gene segments during early B cell development. Analysis of $B$ cells from fetuses and neonates showed that this was not the case in early life $(7,8)$. Rather, Ig gene segment usage in early life is biased toward specific genes, thereby lim- 

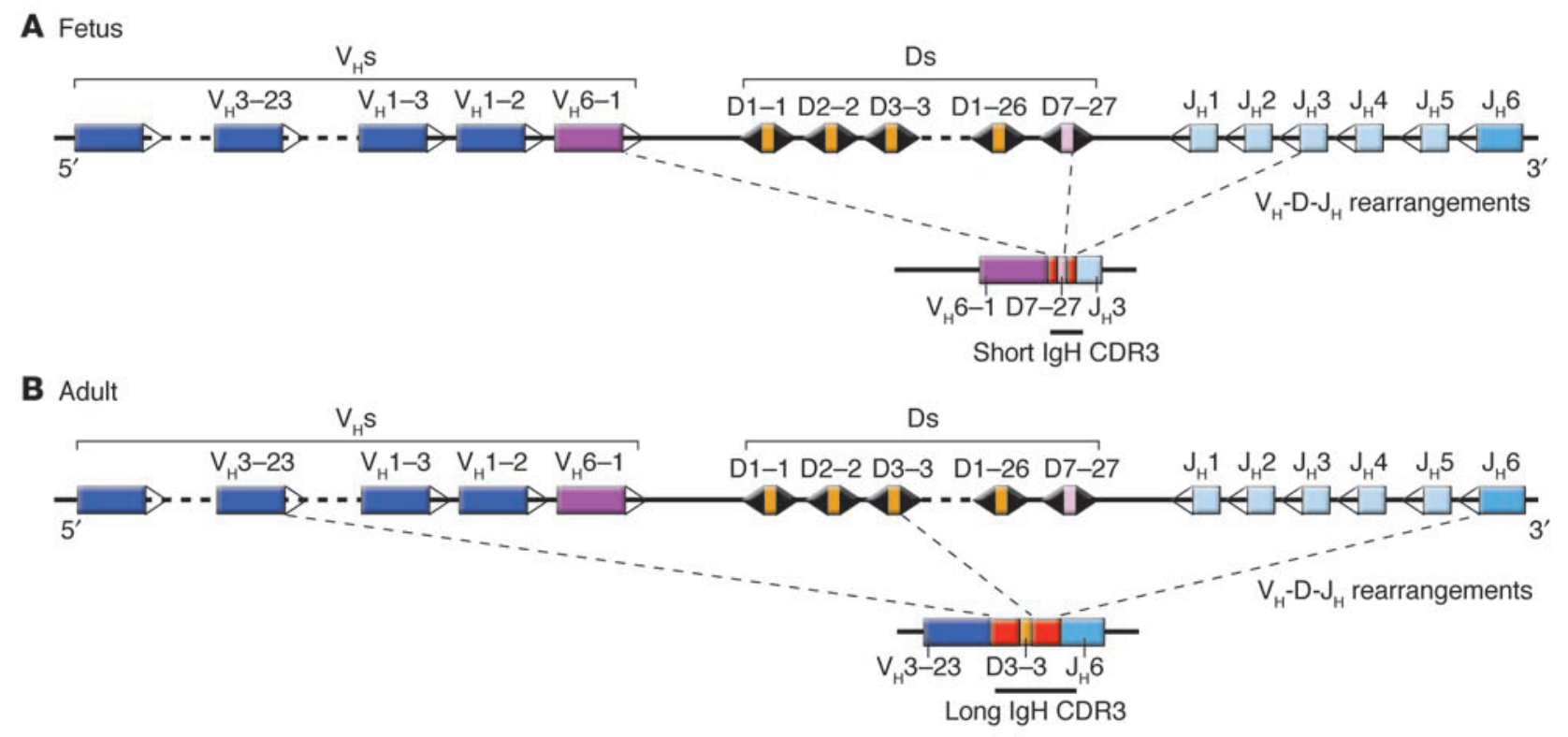

Figure 1

Restricted IgH gene segment recombination during fetal B cell development. Typical V(D)J recombination events in fetal liver (A) and adult bone marrow (B) are depicted. Fetal liver B cells preferentially rearrange variable $V_{H} 6-1$ and diversity D7-27 segments located at the $3^{\prime}$ end of their respectable loci, often combined with the $\mathrm{J}_{\mathrm{H}} 3$ gene segment as shown. The limited nontemplate nucleotide additions between $\mathrm{V}_{\mathrm{H}}-\mathrm{D}$ and $\mathrm{D}$ - $\mathrm{J}_{\mathrm{H}}$ joining regions (red boxes) result in the generation of short IgH CDR3s in early life. Restricted $V(D) J$ recombination during fetal life may result in the production of common natural antibodies recognizing the uniform set of autoantigens identified by Merbl et al. in different individuals (6). In contrast, $B$ cell precursors in adults rarely use $V_{H} 6-1$ and $D 7-27$ segments, but rather use other lg gene segments such as $V_{H} 3-23$ and $D 3-3$, potentially associated with $\mathrm{J}_{\mathrm{H}} 6$ as shown. The extensive nontemplate nucleotide additions (red boxes) may result in the generation of long IgH CDR3s, which — together with diverse V, D, and J segment usage — encode a broader antibody repertoire.

iting the extent of Ig diversity (Figure 1). For example, fetal liver B cells have been shown to have a recombination bias toward heavy chain $(\mathrm{H}) \mathrm{V}_{\mathrm{H}} 6-1$ and D7-27 segments located at the $3^{\prime}$ end of the $\mathrm{V}_{\mathrm{H}}$ and D loci (Figure 1) $(7,8)$. $\mathrm{J}_{\mathrm{H}}$ gene usage is also skewed toward more upstream $\mathrm{J}_{\mathrm{H}} 1$-4 genes, and $\mathrm{J}_{\mathrm{H}} 6$ is rarely found in rearrangements from fetal and neonatal B cells (Figure 1) $(7,8)$. Ig diversity in early life is further limited by low activity of terminal deoxynucleotidyl transferase (TDT), an enzyme that adds nontemplate nucleotides between $\mathrm{V}_{\mathrm{H}^{-}} \mathrm{D}$ and $\mathrm{D}-\mathrm{J}_{\mathrm{H}}$ joining regions $(7,8)$. As a consequence of the lack of insertion of nontemplate nucleotides combined with the fact that D7-27, the segment preferentially used in fetal liver B cells, is the shortest D segment, Ig heavy chain $(\operatorname{IgH})$ complementarity-determining region 3 (CDR3) involved in antigen recognition is particularly short in fetuses and neonates compared with that in adults $(7,8)$.

Taken together, these restrictions to $\mathrm{V}(\mathrm{D}) \mathrm{J}$ recombination that occur during fetal and neonatal life may explain both the generation of $\mathrm{B}$ cell clones that recognize common autoantigens and the similarities in antibody repertoires among different individuals. It is likely that this incomplete fetal/neonatal antibody repertoire is not detrimental to neonates, because protective maternal IgG antibodies cross the placenta and provide potent and properly selected antibodies required for host defense.

\section{B cell tolerance checkpoints in fetuses and neonates}

IgH CDR3s generated by V(D)J recombination play an essential role in conferring polyreactivity to natural antibodies (3). In adults, higher TDT activity and usage of $\mathrm{J}_{\mathrm{H}} 6$ (the longest of all $\mathrm{J}_{\mathrm{H}} \mathrm{s}$ ) are responsible for the production of longer IgH CDR3s than those in fetuses/neonates. In addition, TDTdependent nontemplate nucleotides often encode positively charged amino acids rarely found in D segments, which, when combined with long IgH CDR3s, often lead to the production of autoreactive antibodies $(1,7)$. Although this random $V(D) J$ recombination described in healthy adults generates a large number of autoreactive B cells, such autoreactive clones are counterselected at two major checkpoints $(1,9)$. Central $\mathrm{B}$ cell tolerance is established in the bone marrow at the first checkpoint between the early immature and immature B cell stages, and this silences most polyreactive and anti-nuclear antibody-expressing B cells. A second counterselection step mediates the peripheral B cell tolerance checkpoint and serves to remove autoreactive B cells that recently emigrated from the bone marrow before they enter the long-lived mature naive $\mathrm{B}$ cell pool $(1,9)$. The regulation of central B cell tolerance requires proper B cell receptor (BCR) signaling. Alterations in BCR signaling described in patients with X-linked agammaglobulinemia (XLA) result in a defective central $B$ cell tolerance checkpoint, which is manifested by a failure to counterselect polyreactive developing B cells in these patients (10). Little is known about the molecules and pathways that regulate human peripheral B cell tolerance checkpoints. Our recent studies suggest that $\mathrm{T}$ cells mediate peripheral $\mathrm{B}$ cell tolerance through CD40/CD40 ligand and MHC class II/T cell receptor interactions (E. Meffre, personal communication).

What could account for the production of autoreactive B cells during fetal and neonatal life? The restricted $V(D) J$ recombination in fetuses leads to a limited B cell repertoire, which has been clearly shown by Merbl et al. to contain autoreactive/poly- 
reactive clones (6). Among the neonatal specificities are those encoded by the $\mathrm{V}_{\mathrm{H}} 6$ 1 gene, which is often used during fetal life and has been reported to intrinsically encode anti-single-stranded DNA and anticardiolipin autoreactive antibodies (11). How do such autoreactive clones escape B cell tolerance? Perhaps central and peripheral B cell tolerance checkpoints identified in adults do not yet function during fetal liver or fetal bone marrow B cell development. Differences in the thresholds of BCR signaling may provide a basis for differences in central B cell tolerance checkpoint regulation between fetuses/neonates and adults and could thereby lead to increased production of polyreactive B cells. In addition, the immature fetal/neonatal immune system may lack the necessary "mature" $\mathrm{T}$ cell population that regulates the peripheral B cell tolerance checkpoint, allowing autoreactive/polyreactive $\mathrm{B}$ cells to be recruited in the periphery and secrete natural IgM polyreactive antibodies. Thus, both the mechanisms that generate diversity and those that mediate central and peripheral counterselection in the fetal immune system result in a repertoire of limited diversity and pathogenicity.

\section{Natural antibodies, polyreactive $B$ cells, and autoimmunity}

Natural antibodies are believed to have beneficial functions. They have been shown to be an essential first line of defense against pathogens through either activation of complement or formation of immune complexes that are delivered to follicular dendritic cells in secondary lymphoid organs (reviewed in ref. 12). Natural antibodies also participate in the clearance of apoptotic cells and thereby limit circulation of autoantigens and reduce the risk of autoimmunity. By extension, autoreactive antibodies encoded by the conserved $\mathrm{V}_{\mathrm{H}} 6-1$ gene may help eliminate fetal apoptotic cells and debris containing DNA molecules because those antibodies have anti-nucleic acid reactivity (11). Later in life, autoreactive $V_{H} 6$-1-expressing $B$ cells are rare, but autoreactive clones expressing another intrinsically self-reactive $\mathrm{V}_{\mathrm{H}}, \mathrm{V}_{\mathrm{H}} 4-34$, develop and accumulate in the mature naive $B$ cell compartment. B cells expressing antibodies encoded by $\mathrm{V}_{\mathrm{H}} 4-34$ variable regions recognize I/i carbohydrate self determinants displayed on red blood cells, and such autoantibodies may participate in the clearance of dying erythrocytes (13). Germlineencoded $\mathrm{V}_{\mathrm{H}} 4-34$ antibodies also cross-react with bacterial LPS, suggesting a role in host defense. Despite a potential protective role, the $\mathrm{V}_{\mathrm{H}} 4-34$ gene encodes all pathogenic anti-I/i cold-agglutinin antibodies, indicating that natural antibodies can have damaging effects (13). Indeed, serum natural IgM anti-nonmuscle myosin heavy chain type II (anti-NMHC-II) antibodies have recently been shown to mediate local reperfusion injury after ischemia associated with myocardial infarction, stroke, or surgery (14). Intracytoplasmic NMHC-II molecules are expressed at the cell surface as a result of hypoxic stress, and binding of natural IgM antibodies that recognize these newly exposed self antigens induces complement activation and tissue damage (14).

Whether the limited and selective autoimmunity, termed the immunculus, is protective or whether it represents the substrate on which pathogenic autoantibodies develop will depend upon the effectiveness of checkpoints following birth. Patients with SLE and rheumatoid arthritis display defective central and peripheral $B$ cell tolerance checkpoints that lead to the accumulation of a large number of autoreactive/polyreactive $B$ cells in the mature naive B cell compartment (9). The alteration of these autoreactive/polyreactive B cells through somatic hypermutation and isotype switching may yield clones expressing high-affinity pathogenic autoantibodies. In mice, nucleotide changes in the Ig sequence of an antibody against phosphorylcholine, the dominant hapten on the pneumococcal cell wall, gave rise to antibodies cross-reacting with DNA (15). In healthy adults, $\mathrm{V}_{\mathrm{H}} 4-34$-expressing autoreactive $\mathrm{B}$ cells are excluded from germinal centers and the memory B cell compartment (16). However, in SLE patients, $\mathrm{V}_{\mathrm{H}} 4-34-$ expressing B cells are recruited in germinal centers, where they may produce pathogenic autoantibodies (16). Indeed, such serum $\mathrm{V}_{\mathrm{H}} 4-34$ antibody levels correlate with lupus disease activity (17).

The events that drive breaks in B cell tolerance are unknown, but evidence is emerging that TLRs contribute to activation of autoreactive/polyreactive B cells. TLRs recognize microbial components as well as endogenous self antigens such as DNA and RNA (18). Nuclear autoantigens containing DNA and RNA molecules found in the serum of SLE patients may concomitantly trigger autoreactive/polyreactive BCRs and TLRs and thereby induce the production of autoantibodies binding double-stranded DNA and/or histones as well as nuclear ribonucleoproteins in these patients (19). The role of TLRs during fetal/neonatal B cell ontogeny is unknown. We speculate that TLR binding by self antigens released during developmental apoptosis contributes to activation of polyreactive B cells and secretion of natural antibodies in early human life.

In conclusion, fetal/neonatal autoantibodies are different from adult autoantibodies, especially those associated with autoimmune disease and organ damage. Fetal autoantibodies are IgMs with germline-encoded CDR3s that arise from restricted $V(D) J$ recombination, whereas pathogenic autoantibodies in adults are mostly high-affinity, somatically mutated IgGs that may result from failures of counterselection processes against specific autoantigens. For these reasons, we believe that the immunculus is protective and that defects in the maturation of the immune system later in life lead to abnormal B cell tolerance checkpoints and predispose the individual to autoimmunity.

Address correspondence to: Eric Meffre or Jane E. Salmon, Hospital for Special Surgery, Research Department, 535 E. 70th Street, New York, New York 10021, USA. Phone: (212) 774-2347; Fax: (212) 717-1192; E-mail:meffree@hss.edu (E. Meffre).Phone: (212) 606-1422; Fax: (212) 717-1192; E-mail: salmonj@hss.edu (J.E. Salmon).

1. Wardemann, H., et al. 2003. Predominant autoantibody production by early human B cell precursors. Science. 301:1374-1377.

2. Coutinho, A., Kazatchkine, M.D., and Avrameas, S. 1995. Natural autoantibodies. Curr. Opin. Immunol. 7:812-818.

3. Ichiyoshi, Y., and Casali, P. 1994. Analysis of the structural correlates for antibody polyreactivity by multiple reassortments of chimeric human immunoglobulin heavy and light chain $\mathrm{V}$ segments. J. Exp. Med. 180:885-895.

4. Herzenberg, L.A., et al. 1986. The Ly-1 B cell lineage. Immunol. Rev. 93:81-102.

5. Hayakawa, K., et al. 1999. Positive selection of natural autoreactive B cells. Science. 285:113-116.

6. Merbl, Y., Zucker-Toledano, M., Quintana, F.J., and Cohen, I.R. 2007. Newborn humans manifest autoantibodies to defined self molecules detected by antigen microarray informatics. J. Clin. Invest. 117:712-718. doi:10.1172/JCI29943.

7. Schroeder, H.W., et al. 1995. Developmental regulation of the human antibody repertoire. Ann. N. Y. Acad. Sci. 764:242-262.

8. Souto-Carneiro, M.M., Sims, G.P., Girschik, H., Lee, J., and Lipsky, P.E. 2005. Developmental changes in the human heavy chain CDR3. J. Immunol. 175:7425-7436.

9. Samuels, J., Ng, Y.-S., Coupillaud, C., Paget, D., and Meffre, E. 2005. Human B cell tolerance and its failure in rheumatoid arthritis. Ann. N. Y. Acad. Sci. 1062:116-126.

10. Ng, Y.-S., Wardemann, H., Chelnis, J., Cunningham-Rundles, C., and Meffre, E. 2004. Bruton's 
tyrosine kinase (Btk) is essential for human B cell tolerance. J. Exp. Med. 200:927-934.

11. Logtenberg, T., Young, F.M., van Es, J.H., GmeligMeyling, F.H., and Alt, F.W. 1989. Autoantibodies encoded by the most JH-proximal human immunoglobulin heavy chain variable region gene. J. Exp. Med. 170:1347-1355.

12. Ochsenbein, A.F., and Zinkernagel, R.M. 2000. Natural antibodies and complement link innate and acquired immunity. Immunol. Today. 21:624-630.

13. Pascual, V., et al. 1991. Nucleotide sequence analysis of the $\mathrm{V}$ regions of two IgM cold agglutinins. Evidence that the VH4-21 gene segment is responsible for the major cross-reactive idiotype. J. Immunol. 146:4385-4391.

14. Zhang, M., et al. 2006. Identification of the target self-antigens in reperfusion injury. J. Exp. Med. 203:141-152

15. Ray, S.K., Putterman, C., and Diamond, B. 1996. Pathogenic autoantibodies are routinely generated during the response to foreign antigen: a paradigm for autoimmune disease. Proc. Natl. Acad. Sci.U.S. A. 93:2019-2024.

16. Pugh-Bernard, A.E., et al. 2001. Regulation of inherently autoreactive VH4-34 B cells in the maintenance of human $\mathrm{B}$ cell tolerance.
J. Clin. Invest. 108:1061-1070. doi:10.1172/ JCI200112462.

17. Van Vollenhoven, R.F., et al. 1999. VH4-34 encoded antibodies in systemic lupus erythematosus: a specific diagnostic marker that correlates with clinical disease characteristics. J. Rheumatol. 26:1727-1733

18. Akira, S., Takeda, K., and Kaisho, T. 2001. Tolllike receptors: critical proteins linking innate and acquired immunity. Nat. Immunol. 2:675-680.

19. Marshak-Rothstein, A. 2006. Toll-like receptors in systemic autoimmune disease. Nat. Rev. Immunol. 6:823-835.

\title{
Scavenger receptors clear the air
}

\author{
Edward M. Postlethwait
}

\author{
Department of Environmental Health Sciences, University of Alabama at Birmingham, Birmingham, Alabama, USA.
}

\begin{abstract}
Inhaled environmental oxidants, such as ozone and particulates, have been variably linked to epithelial injury, inflammation, and perturbations in lung development, growth, and function. Reactions between ozone and lung surface lipids likely account for exposure-related pathophysiologic sequelae. In this issue of the JCI, Dahl et al. document a previously unrecognized pulmonary defense against inhaled oxidants in mice: macrophage scavenger receptors (SRs) bind proinflammatory oxidized lipids, thereby decreasing pulmonary inflammation (see the related article beginning on page 757 ). The study adds to our knowledge of diverse lung oxidative processes and identifies a potential regulatory mechanism governing pulmonary inflammation. Further investigations to elucidate more precise mechanisms and to determine the influence of SRs on airway epithelial injury, repair, and remodeling are warranted.
\end{abstract}

\section{Scavenger receptors}

The term scavenger receptor now describes a large family of proteins that feature an unusually broad ligand-binding specificity and are composed of 8 subclasses (1). The first scavenger receptor, scavenger receptor $\mathrm{AI} / \mathrm{II}$ (SR-AI/II) was identified during studies of modified lipoprotein uptake in atherosclerotic plaques (2). It was soon apparent that macrophages (as well as other cell types) bear several forms of scavenger receptors that have both similar and distinct features. The macrophage class A scavenger receptors (SRAs) bind many, but not all, polyanionic mol-

Nonstandard abbreviations used: AM, alveolar macrophage; BAL, bronchoalveolar lavage; ELF, epithelial lining fluid; MARCO, macrophage receptor with collagenous structure; MIP-2, macrophage inflammatory protein-2; PMN, polymorphonuclear leukocyte; PON-GPC, 1-palmitoyl-2-(9'-oxo-nonanoyl)-glycerophosphocholine; RNS, reactive nitrogen species; ROFA, residual oil fly ash; SR, scavenger receptor; SRA, scavenger receptor class A; SR-AI/II, scavenger receptor AI/II.

Conflict of interest: The author has declared that no conflict of interest exists.

Citation for this article: J. Clin. Invest. 117:601-604 (2007). doi:10.1172/JCI31549. ecules including acetylated or oxidized LDL, polyinosinic acid, phosphatidylserine, dextran sulfate, and components of Gram-negative and -positive bacteria (e.g., endotoxin and lipotechoic acid, respectively), prompting one expert to characterize them as "molecular flypaper" (3).

The broad recognition capabilities of scavenger receptors are especially significant to the function of alveolar macrophages (AMs), the primary pulmonary innate immunity sentinel cell. AMs are largely responsible for binding, ingestion, and ultimately clearance of numerous inhaled macromolecules, particles, and pathogens that reach the lower respiratory tract. Previous studies have focused on two SRAs expressed on AMs: the founding member, SR-AI/II, and the more recently described macrophage receptor with collagenous structure (MARCO). SR-AI/II and MARCO knockout mice show increased susceptibility to bacterial pneumonia and more robust inflammatory responses to inhaled environmental particles $(4,5)$, supporting an important role for scavenger receptors in pulmonary innate defense against exogenous challenges. One can envision an evolutionary advantage for scavenger receptor broad recognition because oxidative stress (which occurs under numerous pathologic conditions) initiates lipid oxidation, producing a plethora of reaction/ decomposition products, many of which show biological activities. Thus, if scavenger receptor-lipid binding contributes to bioactive species removal, some degree of nonspecificity in ligand recognition should augment efficacy across a spectrum of lung oxidative and inflammatory processes wherein lipid oxidation occurs.

\section{Pulmonary oxidant challenge}

Ozone and many other inhaled environmental oxidants, including particulates, produce diverse biologic effects that impact both acute and long-term public health. For obvious reasons, long-standing attention has centered on pulmonary sequelae such as physiologic impairments, inflammation, cell injury, and airway remodeling. Epidemiologic and laboratory studies indicate that environmentally relevant exposures may lead to abnormalities in lung growth and development. Importantly, both acute and chronic cardiovascular effects may also occur. In the respiratory tract, the epithelial lining fluid (ELF), which overlays all respiratory tract surfaces, is the first compartment that inhaled materials contact. The physicochemical properties of ozone (high reactivity, poor water solubility) constrain its diffusion through the ELF to the underlying epithelium, so that cellular perturbations likely result from products generated during extracellular (i.e., ELF) reactions $(6,7)$. Because the magnitude of specific biologic responses varies across species, 